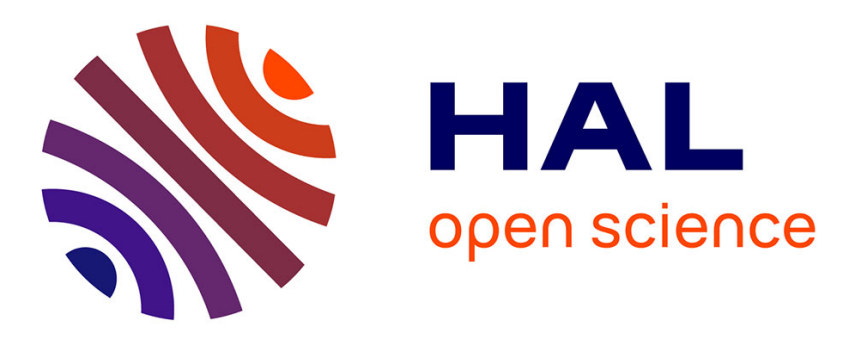

\title{
Anomalies and Vector Space Search: Tools for S-Box Analysis
}

Xavier Bonnetain, Léo Perrin, Shizhu Tian

\section{To cite this version:}

Xavier Bonnetain, Léo Perrin, Shizhu Tian. Anomalies and Vector Space Search: Tools for S-Box Analysis. ASIACRYPT 2019 - 25th International Conference on the Theory and Application of Cryptology and Information Security, Dec 2019, Kobe, Japan. pp.196-223, 10.1007/978-3-030-34578-5_8 . hal-02396738

\section{HAL Id: hal-02396738 \\ https://hal.inria.fr/hal-02396738}

Submitted on 6 Dec 2019

HAL is a multi-disciplinary open access archive for the deposit and dissemination of scientific research documents, whether they are published or not. The documents may come from teaching and research institutions in France or abroad, or from public or private research centers.
L'archive ouverte pluridisciplinaire HAL, est destinée au dépôt et à la diffusion de documents scientifiques de niveau recherche, publiés ou non, émanant des établissements d'enseignement et de recherche français ou étrangers, des laboratoires publics ou privés. 


\title{
Anomalies and Vector Space Search: Tools for S-Box Analysis ${ }^{\star}$
}

\author{
Xavier Bonnetain ${ }^{1,2}$, Léo Perrin ${ }^{1}$ and Shizhu Tian ${ }^{1,3,4}$ \\ 1 Inria, France \\ 2 Sorbonne Université, Collège doctoral \\ 3 State Key Laboratory of Information Security, Institute of Information \\ Engineering, Chinese Academy of Sciences, Beijing, China \\ 4 School of Cyber Security, University of Chinese Academy of Sciences, Beijing, \\ China \\ xavier.bonnetain@inria.fr, leo.perrin@inria.fr, tianshizhu@iie.ac.cn
}

\begin{abstract}
S-boxes are functions with an input so small that the simplest way to specify them is their lookup table (LUT). How can we quantify the distance between the behavior of a given S-box and that of an S-box picked uniformly at random?

To answer this question, we introduce various "anomalies". These real numbers are such that a property with an anomaly equal to $a$ should be found roughly once in a set of $2^{a}$ random S-boxes. First, we present statistical anomalies based on the distribution of the coefficients in the difference distribution table, linear approximation table, and for the first time, the boomerang connectivity table.

We then count the number of S-boxes that have block-cipher like structures to estimate the anomaly associated to those. In order to recover these structures, we show that the most general tool for decomposing S-boxes is an algorithm efficiently listing all the vector spaces of a given dimension contained in a given set, and we present such an algorithm. Combining these approaches, we conclude that all permutations that are actually picked uniformly at random always have essentially the same cryptographic properties and the same lack of structure.
\end{abstract}

Keywords: S-Box, Vector space search, BCT, Shannon effect, Anomaly, Boolean Functions.

\section{Introduction}

S-boxes are small functions with an input small enough that they can be specified by their lookup tables. If $F$ is an S-box with an $n$-bit input then it is feasible to describe it using only the sequence $F(0), F(1), \ldots, F\left(2^{n}-1\right)$ since, in the vast majority of the cases, $3 \leq n \leq 8$. S-boxes can therefore correspond to arbitrarily complex functions. In practice, such components are the only source of nonlinearity of many symmetric primitives. Most prominently, the AES [1] uses an 8-bit bijective S-box.

\footnotetext{
* The full version of this paper is available on eprint (report 2019/528) [10].
} 
The aim of a block cipher is to simulate a pseudo-random permutation (PRP), meaning that it should not be possible for an attacker given a black box access to a block cipher with a secret key and to a permutation picked uniformly at random to figure out which is which. In this context, it might a priori make intuitive sense for block cipher designers to use (pseudo-)random components to design their algorithm. However, this approach would have substantial shortcomings in practice. For example, a random S-box is a priori hard to implement in hardware, random components are unlikely to yield an easy to analyze cipher, their mediocre properties may imply a higher number of rounds (which would slow the cipher down), and the seeds used to generate its random components would have to be published.

Instead, in practice, S-boxes are constructed taking multiple design requirements into account. For example, the mathematical properties of this component can be leveraged to prove that an algorithm is safe from differential [2] or linear [29] cryptanalysis. At the same time, the S-box may be intended to be implemented in hardware or in a bit-sliced fashion, in which case it is necessary to give it a specific structure that will ease such implementations.

While it is easy to compare the properties of two given S-boxes (we can simply compute them and then rank them), it is not trivial to quantify how different they are from an S-box picked uniformly at random with regard to each of their properties. Informally, the comparison with such an "ideal" object will quantify the distance between an S-box and random ones: if the property of the studied S-box is unlikely to occur by chance, then it means that the S-box is much better (or much worse) than average. In this paper, we build upon a framework introduced in [6] to provide both definitions and practical means to compute such probabilities.

Let $\mathfrak{S}_{2^{n}}$ be the set of all $n$-bit permutations and let $F \in \mathfrak{S}_{2^{n}}$. As mentioned above, there are two sets of properties that are relevant when investigating $S$ boxes: how good their cryptographic properties are and whether or not they have some structure. Hence, in order to compare $F$ with a random S-box, we need to be able to answer the following two questions.

1. What is the probability that an S-box picked uniformly in $\mathfrak{S}_{2^{n}}$ has differential/linear properties at least as good as those of $F$ ?

2. How can we recover the structure of $F$ (if it has any)?

Answering the first question can also help us better understand the properties of random permutations and thus to better estimate the advantage of an adversary trying to distinguish a (round-reduced) block cipher from a random permutation.

On the other hand, the second one is related to so-called white-box cryptography, i.e. to implementation techniques that will hide a secret from an attacker with a total access to the implementation of the algorithm. In practice, in order to try and hide for instance an AES key, the attacker will only be given access to an implementation relying on big lookup tables that hide the details of the computations. Recovering the original structure of these tables can also be seen as a particular case of S-box reverse-engineering in the sense of [6]. 


\subsection{Our Contributions}

A Key Concept: Anomalies. We answer the two questions asked above using different variants of a unique approach based on what we call anomalies. Intuitively, an anomaly is a real number that quantifies how unlikely a property is. For example, there are very few differentially- 6 uniform 8-bit permutations, ${ }^{5}$ meaning that the anomaly of this property should be high. However, we could argue that what matters in this case is not just the number of differentially- 6 uniform permutations but the number of permutations with a differential uniformity at most equal to 6 . In light of this, we define anomalies as follows.

Definition 1 (Anomaly). Let $F \in \mathfrak{S}_{2^{n}}$ and let $P$ be a function mapping $\mathfrak{S}_{2^{n}}$ to a partially ordered set. The anomaly of $P(F)$ is defined as $\mathrm{A}(P(F))=$ $-\log _{2}(\operatorname{Pr}[P(G) \leq P(F)])$, where the probability is taken over $G \in \mathfrak{S}_{2^{n}}$. We can equivalently write

$$
\mathrm{A}(P(F))=-\log _{2}\left(\frac{\left|\left\{G \in \mathfrak{S}_{2^{n}}, P(G) \leq P(F)\right\}\right|}{\left|\mathfrak{S}_{2^{n}}\right|}\right) .
$$

The negative anomaly of $P(F)$ is $\overline{\mathrm{A}}(P(F))=-\log _{2}(\operatorname{Pr}[P(G) \geq P(F)])$.

Regardless of $P$, we always have $2^{-\mathrm{A}(P(F))}+2^{-\overline{\mathrm{A}}(P(F))}=1+\operatorname{Pr}[P(G)=P(F)]$.

In the example given above, $P$ is simply the function returning the differential uniformity of a permutation. The anomaly of the differential uniformity then gets higher as the differential uniformity of $F$ decreases under the median differential uniformity as there are fewer permutations with a low differential uniformity. At the same time, the negative anomaly of the differential uniformity increases as the differential uniformity increases above its median value. To put it differently, the anomaly of $P(F)$ quantifies how many S-boxes are at least as good ${ }^{6}$ as $F$ in terms of $P$, and the negative one how many are at least as bad as $F$. In this paper, we study different anomalies and design new tools that allow their estimation for any S-box.

A property with a high anomaly can be seen as distinguisher in the usual sense, i.e. it is a property that differentiates the object studied from one picked uniformly at random. However, unlike usual distinguishers, we do not care about the amount of data needed to estimate the probabilities corresponding to the anomalies.

Statistical Anomalies. In [6] and [34], the notions of "differential" and "linear" anomalies were introduced. Definition 1 is indeed a generalization of them. They are based on properties $P$ that correspond to how good the differential and linear properties are. In Section 2, we generalize this analysis to take into account the corresponding negative anomalies, and we introduce the use of the so-called

\footnotetext{
${ }^{5}$ We formally define differential uniformity later. All that is needed in this discussion is that the differential uniformity is an integer which is better when lower.

${ }^{6}$ In this paper, the properties $P$ considered are better when lower.
} 
Boomerang Connectivity Table (BCT) [17] for this purpose. To this end, we establish the distribution of the coefficients of the BCT of a random permutation. As an added bonus, this new result allows a better estimation of the advantage of an adversary in a boomerang attack.

Structural Anomalies. Anomalies can also be related to the presence of a structure. For example, for $n$-bit Boolean functions, the existence of a simple circuit evaluating a function is unlikely:

"almost all functions" of $n$ arguments have "an almost identical" complexity which is asymptotically equal to the complexity of the most complex function of $n$ arguments.

This statement of Lupanov [28] summarizes the so-called Shannon effect [39]. In other words, the existence of a short description is an unlikely event for a Boolean function. Here, we generalize this observation to permutations of $\mathbb{F}_{2}^{n}$ and construct anomalies that capture how "structured" an S-box is.

In Section 3, we present an estimation of the number of permutations that can be constructed using common S-box generation methods (multiplicative inverse, Feistel networks...) and derive the corresponding anomalies. In order to identify these anomalies, it is necessary to recover said structures when they are unknown. We present a simple approach applicable to inversion-based S-boxes that we successfully apply to the 8-bit S-box of the leaked German cipher Chiasmus. In other cases, we show that the detection of structures with a high anomaly can be performed using a vector space search.

Vector Space Search. We provide an efficient algorithm performing this search: given a set $\mathcal{S}$ of elements of $\{0,1\}^{n}$ and an integer $d$, this algorithm returns all the vector spaces of dimension $d$ that are fully contained in $\mathcal{S}$. We present it in Section 4. While such an algorithm is needed when looking for a structure in an S-box, we expect it to find applications beyond this area.

\subsection{Mathematical Background}

Boolean Functions. Let $\mathbb{F}_{2}=\{0,1\}$. In what follows, we consider the following subsets of the set of all functions mapping $\mathbb{F}_{2}^{n}$ to itself.

- Recall that the set of all $n$-bit permutations is denoted $\mathfrak{S}_{2^{n}}$. It contains $2^{n}$ ! elements. The compositional inverse of $F \in \mathfrak{S}_{2^{n}}$ is denoted $F^{-1}$.

- The set of all $n$-bit linear permutations is denoted $\mathcal{L}_{2^{n}}$. Its size is such that $\left|\mathcal{L}_{2^{n}}\right|=\prod_{i=0}^{n-1}\left(2^{n}-2^{i}\right)$.

For elements of $\mathbb{F}_{2}^{n}$, "+" denotes the characteristic- 2 addition, i.e. the XOR. In cases that might be ambiguous, we use " $\oplus$ " to denote this operation.

Let $F \in \mathfrak{S}_{2^{n}}$ be an S-box. Many of its cryptographic properties can be described using $2^{n} \times 2^{n}$ tables: the LAT, DDT and BCT. They are defined below. 
The Linear Approximation Table (LAT) of $F$ is the table $\mathcal{W}_{F}$ with coefficients $\mathcal{W}_{F}(a, b)=\sum_{x \in \mathbb{F}_{2}^{n}}(-1)^{a \cdot x+b \cdot F(x)}$ where $x \cdot y=\bigoplus_{i=0}^{n-1} x_{i} \times y_{i}$ is the scalar product of two elements $x=\left(x_{0}, \ldots, x_{n-1}\right), y=\left(y_{0}, \ldots, y_{n-1}\right) \in \mathbb{F}_{2}^{n}$. Its maximum for $b \neq 0$ is the linearity of $F$ and is denoted $\ell(F)$. The LAT is used to study linear cryptanalysis $[40,29]$. The set of the coordinates of the coefficients equal to 0 plays a special role, as shown in [15]. It is called the Walsh zeroes of $F$ and is denoted $\mathcal{Z}_{F}=\left\{(a, b) \in\left(\mathbb{F}_{2}^{n}\right)^{2} \mid \mathcal{W}_{F}(a, b)=0\right\} \cup\{(0,0)\}$.

The Difference Distribution Table (DDT) of $F$ is the table $\delta_{F}$ with coefficients $\delta_{F}(a, b)=\#\left\{x \in \mathbb{F}_{2}^{n}, F(x+a)+F(x)=b\right\}$. Its maximum for $a \neq 0$ is the differential uniformity of $F$ and is denoted $u(F)$. The DDT is needed to study differential cryptanalysis [3].

Recently, Cid et al. introduced a new tool which they called Boomerang Connectivity Table (BCT) [17]. It is again a $2^{n} \times 2^{n}$ table $\mathcal{B}_{F}$ defined by

$$
\mathcal{B}_{F}(a, b)=\#\left\{x \in \mathbb{F}_{2}^{n}, F^{-1}(F(x)+b)+F^{-1}(F(x+a)+b)=a\right\} .
$$

Its maximum value for $a, b \neq 0$ is the boomerang uniformity of $F$ and is denoted $\beta_{F}$. As hinted by its name, the BCT is relevant when studying boomerang attacks [41]. Unlike the DDT and LAT, it is necessary that $F$ is a permutation for the BCT to be well defined.

Statistics. Some of our results rely on both the binomial and Poisson distribution. We denote with $\operatorname{Binomial}(n, p)$ the binomial distribution with parameters $p$ and $n$ which correspond respectively to the probability of an event and to the number of trial. It is defined as follows:

$$
\operatorname{Pr}[X=i]=\operatorname{Binomial}(i ; n, p)=p^{i}(1-p)^{n-i}\left(\begin{array}{c}
n \\
i
\end{array}\right) .
$$

It has a mean equal to $n p$ and a variance of $n p(1-p)$. The Poisson distribution with parameter $\lambda$ is defined by

$$
\operatorname{Pr}[X=i]=\operatorname{Poisson}(i ; \lambda)=\frac{e^{-\lambda} \lambda^{i}}{i !}
$$

The mean value and variance of this distribution are both $\lambda$. A binomial distribution with small $p$ can be closely approximated by a Poisson distribution with $\lambda=n p$.

\section{Statistical Properties}

Let us consider a permutation $F$ that is picked uniformly at random from $\mathfrak{S}_{2^{n}}$ and let us consider one of its tables, i.e. its DDT, LAT or BCT. The coefficients in this table may be connected to one another: for example the sum of the coefficients in a row of the DDT have to sum to $2^{n}$. Yet, in practice, the coefficients act like independent and identically distributed random variables. In 
Section 2.1), we recall what the distributions of the DDT and LAT coefficients are and we establish the distribution of the BCT coefficients.

Then, Section 2.2 presents how the knowledge of these distributions can be used to bound the probability that a random permutation has differential/linear/boomerang properties at least as good as those of the S-box investigated. Additionally, we explain in Section 2.3 how our newly gained knowledge of the distribution of the BCT coefficients allows a better estimation of the advantage of the attacker in a boomerang attack.

\subsection{Coefficient Distributions}

In [18], the authors established and experimentally verified the distribution followed by the DDT and LAT coefficients. The distribution of the LAT coefficients was first established in [33] and then provided a different expression in [18]. A more thorough study of the DDT coefficient can be found in [32]. We recall these results in the following two theorems.

Proposition 1 (DDT coefficient distribution [18]). The coefficients in the DDT of a random $S$-Box of $\mathfrak{S}_{2^{n}}$ with $n \geq 5$ are independent and identically distributed random variables following a Poisson distribution Poisson $\left(2^{-1}\right)$.

Proposition 2 (LAT coefficient distribution $[33,18]$ ). The coefficients in the LAT of a random permutation ${ }^{7}$ of $\mathfrak{S}_{2^{n}}$ are independent and identically distributed random variables with the following probability distribution:

$$
\operatorname{Pr}\left[\mathcal{W}_{F}(i, j)=4 z\right]=\frac{\left(\begin{array}{c}
2^{n-1} \\
2^{n-2}+z
\end{array}\right)^{2}}{\left(\begin{array}{c}
2^{n} \\
2^{n-1}
\end{array}\right)}
$$

The situation is the same for the BCT. In order to establish the distribution of the non-trivial coefficients of the $\mathrm{BCT}$ of a random permutation, we first recall an alternative definition of the BCT that was introduced in [26].

Proposition 3 (Alternative BCT definition [26]). Let $F \in \mathfrak{S}_{2^{n}}$ be a permutation. For any $a, b \in \mathbb{F}_{2}^{n}$, the entry $\mathcal{B}_{F}(a, b)$ of the $B C T$ of $F$ is given by the number of solutions in $\mathbb{F}_{2}^{n} \times \mathbb{F}_{2}^{n}$ of the following system of equations

$$
\left\{\begin{array}{l}
F^{-1}(x+b)+F^{-1}(y+b)=a \\
F^{-1}(x)+F^{-1}(y)=a
\end{array}\right.
$$

We use this theorem to obtain the distribution of the coefficients in the BCT.

Theorem 1 (BCT coefficient distribution). If $F$ is picked uniformly at random in $\mathfrak{S}_{2^{n}}$, then its coefficients with $a, b \neq 0$ can be modeled like independent and identically distributed random variables with the following distribution:

$$
\operatorname{Pr}\left[\mathcal{B}_{F}(a, b)=c\right]=\sum_{2 i_{1}+4 i_{2}=c} P_{1}\left(i_{1}\right) P_{2}\left(i_{2}\right),
$$

\footnotetext{
7 The distribution of the coefficients in the LAT of random functions (not permuta-
} tions) is also provided in [18]. 
where $P_{1}$ and $P_{2}$ are stochastic variable following binomial distributions: $P_{1}(i)=$ Binomial $\left(i ; 2^{n-1}, \frac{1}{2^{n}-1}\right)$ and $P_{2}(i)=\operatorname{Binomial}\left(i ; 2^{2 n-2}-2^{n-1}, \frac{1}{\left(2^{n}-1\right)^{2}}\right)$.

Proof. For any $x, y \in \mathbb{F}_{2}^{n}$ such that $x \neq y$, we define

$$
S_{x, y}=\{(x, y),(y, x),(x+b, y+b),(y+b, x+b)\},
$$

which is of cardinality 4 unless $x+y=b$, in which case it only contains 2 elements. These sets are such that a pair $(x, y)$ is a solution of System (1) if and only if all the elements in $S_{x, y}$ are as well. In order to prove this theorem, we will partition the set of all pairs of elements of $\mathbb{F}_{2}^{n}$ into such sets $S_{x, y}$.

To this end, we consider the following equivalence relation: $(x, y) \sim\left(x^{\prime}, y^{\prime}\right)$ if and only if the multisets $S_{x, y}$ and $S_{x^{\prime}, y^{\prime}}$ are identical. The corresponding equivalence classes are of size 4 except when $x+y=b$, in which case they contain only 2 elements. There are in total $2^{n-1}$ classes of size 2 . As there are $2^{n}\left(2^{n}-1\right)$ ordered pairs of elements in $\mathbb{F}_{2}^{n}$, we deduce that there are $\left(2^{n}\left(2^{n}-1\right)-2 \times 2^{n-1}\right) / 4$ classes of cardinality 4 , i.e. $2^{2 n-2}-2^{n-1}$.

Then, in order for System (1) to have exactly $c$ solutions, we need that there exists $i_{1}$ solutions in classes of size 4 and $i_{2}$ in classes of size 2 , where $2 i_{1}+4 i_{2}=c$. We deduce that

$$
\operatorname{Pr}\left[\mathcal{B}_{F}(a, b)=c\right]=\sum_{2 i_{1}+4 i_{2}=c} P_{1}\left(i_{1}\right) P_{2}\left(i_{2}\right),
$$

where $P_{1}\left(i_{1}\right)$ (respectively $P_{2}\left(i_{2}\right)$ ) is the probability that there exists $i_{1}$ classes of size 4 (resp. 2) that are solutions of System (1). Let us now prove that the distributions of $P_{1}\left(i_{1}\right)$ and $P_{2}\left(i_{2}\right)$ are as stated in the theorem.

Size 2. In this case, it holds that $y=x+b$ so that the lines of System (1) are identical. We assume that $F^{-1}(x)+F^{-1}(x+b)=a$ holds with probability $1 /\left(2^{n}-1\right)$ as $F^{-1}(x)+F^{-1}(x+b)$ can take any value in $\mathbb{F}_{2}^{n} \backslash\{0\}$. Since there are $2^{n-1}$ such pairs, $P_{1}\left(i_{1}\right)$ corresponds to a binomial distribution with $2^{n-1}$ repetitions of a Bernoulli trial that succeeds with probability $\left(2^{n}-1\right)^{-1}$.

Size 4. The two equations of System (1) are now independent. Using the same reasoning as above, we assume that each line holds with probability $1 /\left(2^{n}-\right.$ $1)$. Since there are $2^{2 n-2}-2^{n-1}$ such pairs, $P_{2}\left(i_{2}\right)$ corresponds to a binomial distribution with parameters $2^{2 n-2}-2^{n-1}$ and $\left(2^{n}-1\right)^{-2}$.

\subsection{Anomalies in Table Coefficients Distributions}

Building upon the general approach presented in [6], we can define several anomalies using the distribution of the coefficients in the tables of a permutation $F \in \mathfrak{S}_{2^{n}}$. We will then be able to estimate the values of the corresponding anomalies using the distributions derived in the previous section. 
Maximum Value. For any table, the maximum absolute value of all coefficients is a natural property to use to construct an anomaly as the integers are ordered. Let $\max _{T}: \mathfrak{S}_{2^{n}} \rightarrow \mathbb{N}$ be the function mapping a permutation $F \in \mathfrak{S}_{2^{n}}$ to the maximum absolute value of the non-trivial coefficients in a table $T$. Then we can use the distributions in Propositions 1 and 2 as well as Theorem 1 to estimate the associated anomalies:

$$
\mathrm{A}\left(\max _{T}(F)\right)=-\left(2^{n}-1\right)^{2} \log _{2}\left(\sum_{i=0}^{\max _{T}(F)} p_{i}\right)
$$

where $p_{i}$ is the probability that $T(a, b)=i$. Indeed, there are only $\left(2^{n}-1\right)^{2}$ non-trivial coefficients in the DDT, LAT and BCT as the first row and column are fixed in each case. The (negative) anomalies corresponding to the differential uniformity, linearity and boomerang uniformity for $n=8$ are given in the appendix of the full version of this paper [10].

Maximum Value and Number of Occurrences. In $\mathfrak{S}_{2^{8}}$, the anomaly of a differential uniformity of 8 is equal to 16.2 but, for a differential uniformity of 6 , it is 164.5. In order to have a finer grained estimate of how unlikely the properties of an S-box are, we combine the maximum coefficient in one of its tables with its number of occurrences as was first done in [6]. For a $2^{n} \times 2^{n}$ table of integers $T$, let $\mathrm{MO}$ be the function such that $\operatorname{MO}(T)=(c, m)$ where $c$ is the maximum absolute value in $T$ and $m$ is its number of occurrences (where the first row and column are ignored). The set $\mathbb{N} \times \mathbb{N}$ in which the output of $\mathrm{MO}$ lives can be ordered using the lexicographic ordering, i.e. $(x, y) \leq\left(x^{\prime}, y^{\prime}\right)$ if and only if $x<x^{\prime}$ or $x=x^{\prime}$ and $y \leq y^{\prime}$. We then define the differential, linear and boomerang anomalies of $F$ as respectively

$$
\left.\mathrm{A}^{\mathrm{d}}(F)=\mathrm{A}\left(\mathrm{MO}\left(\delta_{F}\right)\right)\right), \mathrm{A}^{\ell}(F)=\mathrm{A}\left(\mathrm{MO}\left(\mathcal{W}_{F}\right)\right), \text { and } \mathrm{A}^{\mathrm{b}}(F)=\mathrm{A}\left(\mathrm{MO}\left(\mathcal{B}_{F}\right)\right)
$$

This definition of the differential and linear anomalies matches with the one given in [34]. The boomerang anomaly was not used before. We also introduce the negative differential, linear and boomerang anomalies as the corresponding negative anomalies.

We estimate these anomalies for a table $T$ using the following expression:

$$
\mathrm{A}(\mathrm{MO}(T) \leq(c, m))=-\log _{2}\left(\sum_{k=0}^{m}\left(\begin{array}{c}
\left(2^{n}-1\right)^{2} \\
k
\end{array}\right) \times p_{c}^{k} \times\left(\sum_{j=0}^{c-1} p_{j}\right)^{\left(2^{n}-1\right)^{2}-k}\right)
$$

where $p_{i}$ is the probability that $T(a, b)=|i|$. For the corresponding negative anomaly, we use the following relation:

$$
2^{\mathrm{A}(\mathrm{MO}(T) \leq(c, m))}+2^{\overline{\mathrm{A}}(\mathrm{MO}(T) \leq(c, m))}=1+\left(\begin{array}{c}
\left(2^{n}-1\right)^{2} \\
m
\end{array}\right) p_{c}^{m}\left(\sum_{j=0}^{c-1} p_{j}\right)^{\left(2^{n}-1\right)^{2}-m} .
$$




\subsection{Tighter Advantage Estimations for Boomerang Attacks}

The coefficient distribution we established in Theorem 1 can also be used to compute the expected value of a BCT coefficient. This in turn implies a better understanding of the advantage an adversary has in a boomerang attack.

Theorem 2. The expected value for each BCT coefficient of a random permutation of $\mathfrak{S}_{2^{n}}$ converges towards 2 as $n$ increases.

Proof. Let $F \in \mathfrak{S}_{2^{n}}$ be picked uniformly at random. The expected value $E$ of $\mathcal{B}_{F}(a, b)$ is $\sum_{c=0}^{2^{n}} \operatorname{Pr}\left[\mathcal{B}_{F}(a, b)=c\right] c$. Using Theorem 1, we express $\operatorname{Pr}\left[\mathcal{B}_{F}(a, b)=c\right]$ using two binomial distributions $P_{1}$ and $P_{2}$ so that

$$
\begin{aligned}
E & =\sum_{c=0}^{2^{n}} c \times\left(\sum_{2 i_{1}+4 i_{2}=c} P_{1}\left(i_{1}\right) P_{2}\left(i_{2}\right)\right) \\
& =\sum_{c=0}^{2^{n}} \sum_{i_{1}=0}^{2^{n-1}} \sum_{i_{2}=0}^{2^{n-2}}\left(2 i_{1}+4 i_{2}\right) P_{1}\left(i_{1}\right) P_{2}\left(i_{2}\right) \times\left[2 i_{1}+4 i_{2}=c\right],
\end{aligned}
$$

where the expression between the brackets is equal to 1 if $2 i_{1}+4 i_{2}=c$, and 0 otherwise. Reordering the sums, we obtain the following expected value:

$$
E=\underbrace{\sum_{i_{1}=0}^{2^{n-1}} \sum_{i_{2}=0}^{2^{n-2}}\left(2 i_{1}+4 i_{2}\right) P_{1}\left(i_{1}\right) P_{2}\left(i_{2}\right)}_{E(n)} \underbrace{\sum_{c=0}^{2^{n}}\left[2 i_{1}+4 i_{2}=c\right]}_{\leq 1} .
$$

We then approximate the binomial distributions $P_{1}$ and $P_{2}$ by Poisson distributions, namely $P_{1}(i) \approx \operatorname{Poisson}\left(i ; 2^{-1}\right)=e^{-\frac{1}{2}} 2^{-i} /(i !)$ and $P_{1}(i) \approx \operatorname{Poisson}\left(i ; 4^{-1}\right)=$ $e^{-\frac{1}{4}} 4^{-i} /(i$ !). We get

$$
\begin{aligned}
E(n) & =\sum_{i_{1}=0}^{2^{n-1}} \sum_{i_{2}=0}^{2^{n-2}}\left(2 i_{1}+4 i_{2}\right) \frac{e^{-\frac{1}{2}} 2^{-i_{1}}}{i_{1} !} \frac{e^{-\frac{1}{4}} 4^{-i_{2}}}{i_{2} !} \\
& =\sum_{i_{1}=1}^{2^{n-1}} \frac{e^{-\frac{1}{2}}\left(\frac{1}{2}\right)^{i_{1}-1}}{\left(i_{1}-1\right) !} \sum_{i_{2}=0}^{2^{n-2}} \frac{e^{-\frac{1}{4}}\left(\frac{1}{4}\right)^{i_{2}}}{i_{2} !}+\sum_{i_{1}=0}^{2^{n-1}} \frac{e^{-\frac{1}{2}}\left(\frac{1}{2}\right)^{i_{1}}}{i_{1} !} \sum_{i_{2}=1}^{2^{n-2}} \frac{e^{-\frac{1}{4}}\left(\frac{1}{4}\right)^{i_{2}-1}}{\left(i_{2}-1\right) !} .
\end{aligned}
$$

As all sums converge towards 1 as $n$ increases, the limit of $E(n)$ is 2 . On the other hand, we remark that $E \leq E(n)$ because of Equation (2), and that

$$
E \geq \sum_{i_{1}=0}^{2^{n-2}} \sum_{i_{2}=0}^{2^{n-3}}\left(2 i_{1}+4 i_{2}\right) P_{1}\left(i_{1}\right) P_{2}\left(i_{2}\right) \underbrace{\sum_{c=0}^{2^{n}}\left[2 i_{1}+4 i_{2}=c\right]}_{=1}=E(n-1),
$$

so $E(n-1) \leq E \leq E(n)$. As $E(n)$ converges to 2 as $n$ increases, so does $E$.

The expected probability of a boomerang characteristic $E_{k}^{-1}\left(E_{k}(x) \oplus b\right) \oplus$ $E_{k}^{-1}\left(E_{k}(x \oplus a) \oplus b\right)=a$ is thus $2^{1-n}$ and not $2^{-n}$ as we might expect. 


\subsection{Experimental Results}

Verification. To check the validity of our approach to estimate the statistical anomalies, we picked $2^{21}$ permutations from $\mathfrak{S}_{2^{8}}$ uniformly at random. We then counted the number $N_{t}$ of permutations $F$ such that $\lfloor\mathrm{A}(F)\rfloor=t$, and we obtained the following results (only anomalies above 19 are listed):

$$
\begin{array}{ll}
\mathrm{A}^{\ell}(F): N_{19}=1, N_{21}=1 & \overline{\mathrm{A}}^{\ell}(F): N_{19}=1 \\
\mathrm{~A}^{\mathrm{d}}(F): \text { See below } & \overline{\mathrm{A}^{\mathrm{d}}(F): N_{20}=1} \\
\mathrm{~A}^{\mathrm{b}}(F): N_{19}=3 & \overline{\mathrm{A}}^{\mathrm{b}}(F): N_{20}=2 .
\end{array}
$$

We deduce that the anomalies other than $\mathrm{A}^{\mathrm{d}}(F)$ behave as we expect: in a set of size $2^{t}$, we can expect to see about 1 permutation with an anomaly of $t$.

However, for $\mathrm{A}^{\mathrm{d}}(F)$, our results do not quite match the theory. Indeed, we have found too many permutations with a high differential anomaly for it to be a coincidence:

$$
\begin{aligned}
\mathrm{A}^{\mathrm{d}}(F): & N_{19}=7, N_{20}=8, N_{21}=2, N_{22}=1, N_{23}=2, \\
& N_{24}=1, N_{25}=1, N_{26}=1, N_{28}=1 .
\end{aligned}
$$

Recall that our estimates of the table-based anomalies rely on the assumption that the coefficients behave like independent random variables. While we experimentally found this assumption to yield accurate models in practice for all tables, it fails to accurately predict the behavior of the maximum value and its number of occurrences in the case of the DDT.

S-boxes from the Literature. We computed the statistical anomalies we defined above for several 8-bit S-boxes from the literature that we obtained from [36]. The results are given in Table 1 . We also list the number $N_{V}$ of vector spaces of dimension $n$ contained in $\mathcal{Z}_{s}$; its importance will appear later in Section 3.

The statistical anomalies of the AES S-box, i.e. of the multiplicative inverse, are unsurprisingly very large. But they are too large: an anomaly cannot be higher than $\log _{2}\left(\left|\mathfrak{S}_{2^{n}}\right|\right)$. Our estimates do not hold for objects with properties as extreme as those of the inverse.

We can derive other results from this table. For example, 2-round SPNs have a high negative boomerang anomaly but 3-round ones loose this property. Classical 3-round Feistel networks, as used in ZUC_S0, have a boomerang uniformity which is maximum [12] so it is not surprising to see that they have a boomerang anomaly so high that we could not compute it. Even though the S-box of Zorro has a modified Feistel structure (it uses a sophisticated bit permutation rather than a branch swap), it still has a high negative boomerang anomaly.

As expected, the S-boxes that were generated using a random procedure have low positive and negative statistical anomalies. The S-box of MD2 was obtained using the digits of $\pi$, that of the newDES from the American declaration of independence, and that of Turing from the string "Alan Turing".

The correlation between the different statistical anomalies seems complex. On the one hand, there are S-boxes with very different linear and differential 


\begin{tabular}{|c|c|c|c|c|c|c|c|c|}
\hline Type & Cipher & $\mathrm{A}^{\mathrm{d}}(s)$ & $\overline{\mathrm{A}}^{\mathrm{d}}(s)$ & $\mathrm{A}^{\ell}(s)$ & $\overline{\mathrm{A}}^{\ell}(s)$ & $\mathrm{A}^{\mathrm{b}}(s)$ & $\overline{\mathrm{A}}^{\mathrm{b}}(s)$ & $N_{V}(s)$ \\
\hline Inverse & AES & 7382.13 & 0.00 & 3329.43 & 0.00 & 9000.05 & 0.00 & 2 \\
\hline Logarithm & BelT & 74.79 & 0.00 & 122.97 & 0.00 & 0.98 & 0.40 & 2 \\
\hline TKlog & Kuznyechik & 80.63 & 0.00 & 34.35 & 0.00 & 14.18 & 0.00 & 3 \\
\hline \multirow{4}{*}{ SPN (2S) } & CLEFIA_S0 & 2.56 & 0.19 & 25.62 & 0.00 & 0.00 & 15.60 & 6 \\
\hline & Enocoro & 1.92 & 0.36 & 3.26 & 0.15 & 0.00 & 15.60 & 6 \\
\hline & Twofish_p0 & 1.36 & 0.70 & 3.16 & 0.17 & 0.00 & 33.84 & 6 \\
\hline & Twofish_p1 & 1.34 & 0.72 & 3.16 & 0.17 & 0.00 & 25.82 & 6 \\
\hline \multirow{2}{*}{ SPN (3S) } & Iceberg & 17.15 & 0.00 & 3.58 & 0.10 & 0.02 & 3.87 & 2 \\
\hline & Khazad & 16.94 & 0.00 & 3.16 & 0.17 & 0.98 & 0.40 & 2 \\
\hline \multirow{2}{*}{ Feistel } & Zorro & 2.19 & 0.27 & 3.37 & 0.13 & 0.00 & 25.82 & 2 \\
\hline & ZUC_S0 & 16.15 & 0.00 & 3.16 & 0.17 & 0.00 & $\mathrm{NaN}$ & 368 \\
\hline \multirow{4}{*}{ Hill climbing } & Kalyna_pi0 & 104.22 & 0.00 & 235.77 & 0.00 & 29.67 & 0.00 & 2 \\
\hline & Kalyna_pi1 & 122.64 & 0.00 & 268.07 & 0.00 & 29.67 & 0.00 & 2 \\
\hline & Kalyna_pi2 & 129.87 & 0.00 & 239.28 & 0.00 & 5.99 & 0.00 & 2 \\
\hline & Kalyna_pi3 & 122.64 & 0.00 & 242.92 & 0.00 & 26.44 & 0.00 & 2 \\
\hline \multirow{3}{*}{ Random } & Turing & 0.18 & 1.94 & 1.84 & 0.17 & 0.98 & 0.40 & 2 \\
\hline & MD2 & 1.36 & 0.70 & 0.10 & 2.41 & 0.98 & 0.40 & 2 \\
\hline & newDES & 0.44 & 0.73 & 0.32 & 1.95 & 0.14 & 1.86 & 2 \\
\hline Unknown & Skipjack & 0.18 & 1.94 & 54.38 & 0.00 & 0.98 & 0.40 & 2 \\
\hline
\end{tabular}

Table 1: The statistical anomalies and number of vector spaces for some S-boxes from the literature.

anomalies despite the fact that the square of the LAT coefficients corresponds to the Fourier transform of the DDT (see e.g. Skipjack). As evidenced by the anomalies of the S-boxes of Kalyna, which were obtained using a hill climbing method optimizing the differential and linear properties [25], these improvements lead to an observable increase of the boomerang anomaly but it can be marginal.

\section{$3 \quad$ Identifying Structures}

In this section, we go through the most common S-box structures, and present for each of them the density of the set of such S-boxes (up to affine-equivalence) and the methods that can be used to identify them. In practice, S-boxes operating on at least 6 bits usually fall into two categories: those that are based on the inverse in the finite field $\mathbb{F}_{2^{n}}$, and those using block cipher structures.

In both cases, the permutations are usually composed with affine permutations. In the context of white-box cryptography, it is common to compose functions with secret affine permutations so as to obfuscate the logic of the operations used. Hence, for both decomposing S-boxes and attacking white-box implementation, it is necessary to be able to remove these affine layers.

While recovering a monomial structure is simple even when it is masked by affine permutations (see Section 3.1 and our results on the S-box of Chiasmus), it is not the case with block cipher structures. In this section, we show how the the recovery of the pattern used in [7] to remove the affine layers of the Russian S-box can be efficiently automatized (Section 3.2), and applied to both SPNs (Section 3.3) and Feistel network (Section 3.4). The core algorithm needed for 
these attacks is one returning all the vector spaces contained in a set of elements of $\mathbb{F}_{2}^{n}$. We will present such an algorithm in Section 4 .

These techniques allow us to identify the structural anomalies in S-boxes. In order to estimate the anomaly associated with each structure, we upper bound the number of permutation that can be built using each of those that we consider. The corresponding anomalies are summarized in Section 3.5.

\subsection{Multiplicative Inverse}

Such permutations have a very simple structure: there exists two affine permutations $A: \mathbb{F}_{2}^{n} \rightarrow \mathbb{F}_{2^{n}}$ and $B: \mathbb{F}_{2^{n}} \rightarrow \mathbb{F}_{2}^{n}$ such that the permutations $F$ can be written $F=B \circ G \circ A$, where $G$ is the permutation of $\mathbb{F}_{2^{n}}$ defined by $G(x)=x^{2^{n}-2}$. Their use was introduced in [31]; the AES [1] uses such an S-box.

In practice, the implementation of $G$ requires the use of an encoding of the elements of $\mathbb{F}_{2^{n}}$ as elements of $\mathbb{F}_{2}^{n}$. Usually, it is achieved by mapping $x=$ $\left(x_{0}, \ldots, x_{n-1}\right) \in \mathbb{F}_{2}^{n}$ to $\sum_{i=0}^{n-1} x_{i} \alpha^{i}$, where $\alpha \in \mathbb{F}_{2^{n}}$ is the root of an irreducible polynomial with coefficients in $\mathbb{F}_{2}$ of degree $n$. However, this encoding can be seen as being part of $A$ and $B$.

Density of the set. There is only one function $x \mapsto x^{2^{n}-2}$. However, there are fewer than $\left(\left|\mathcal{L}_{2^{n}}\right| 2^{n}\right)^{2}$ distinct permutations affine-equivalent to it. Indeed, $(x \times$ $m)^{2^{n}-2}=x^{2^{n}-2} \times m^{2^{n}-2}$, meaning that for a given pair $(A, B)$ of permutations of $\mathcal{L}_{2^{n}}$ we can define $2^{n}-1$ pairs $\left(A_{i}, B_{i}\right) \in\left(\mathcal{L}_{2^{n}}\right)^{2}$ such that $B_{i} \circ G \circ A_{i}=B_{j} \circ G \circ A_{j}$ for all $i, j$. The same reasoning applies to the Frobenius automorphisms because $\left(x^{2^{i}}\right)^{2^{n}-2}=\left(x^{2^{n}-2}\right)^{2^{i}}$. In the end, there are at most

$$
\underbrace{\left|\mathcal{L}_{2^{n}}\right|^{2}}_{L_{A} \text { and } L_{B}} \times \underbrace{2^{2 n}}_{c_{A} \text { and } c_{B}} \times \underbrace{\left(2^{n}-1\right)}_{\text {multiplication }} \times \underbrace{n}_{\text {Frobenius }}=\frac{2^{n}}{n} \times\left(\left|\mathcal{L}_{2^{n}}\right|\right)^{2}
$$

distinct permutations affine-equivalent to the multiplicative inverse.

How to recognize them? The Chinese cipher SMS4 [20] uses an 8-bit S-box whose structure was not explained. This prompted Liu et al. to try and recover said structure [27]. They successfully identified it as being affine equivalent to the multiplicative inverse using an ad hoc method.

There is a simple test that can be applied to check if a permutation is affineequivalent to the multiplicative inverse when the input/output size is even.

Lemma 1. Let $s: x \mapsto x^{2^{n}-2}$ be a permutation of $\mathbb{F}_{2^{n}}$ and $F \in \mathfrak{S}_{2^{n}}$ with $n$ even be such that $F=B \circ s \circ A$ where $A: x \mapsto L_{A}(x)+c_{A}$ and $B: x \mapsto L_{B}(x)+c_{B}$ are affine permutations. Let $\left\{\left(a_{i}, b_{i}\right)\right\}$ be the set of all coordinates such that $\delta_{F}\left(a_{i}, b_{i}\right)=4$. Then it holds that $b_{i}=L_{B}\left(L_{A}\left(a_{i}\right)^{2^{n}-2}\right)$ for all $i$, meaning that $a_{i} \mapsto b_{i}$ and $s$ are identical up to translations.

Proof. We have that $(x+a)^{e}+x^{e}=b$ has as many solutions as $(y+1)^{e}+$ $y^{e}=b / a^{e}$, meaning that all rows of its DDT contain the same coefficients: 
$\delta_{s}(a, b)=\delta_{s}\left(1, b / a^{e}\right)$. In the case of the inverse for $n$ even, $\delta_{s}(1, c) \in\{0,2\}$ for all $c \neq 1$ and $\delta_{s}(1,1)=4$. Such a function was called locally- $A P N$ in [9].

In our case, we have that $\delta_{F}(a, b)=\delta_{s}\left(L_{A}(a), L_{B}^{-1}(b)\right)$. Using the property we just established with $e=2^{n}-2$, we get $\delta_{F}(a, b)=\delta_{s}\left(1, L_{B}^{-1}(b) /\left(L_{A}(a)\right)^{2^{n}-2}\right)$, where the second coordinate simplifies into $L_{B}^{-1}(b) \times L_{A}(a)$. As a consequence, $\delta_{F}(a, b)=4$ if and only if $L_{B}^{-1}(b)=\left(L_{A}(a)\right)^{2^{n}-2}$, which is equivalent to $b=$ $L_{B}\left(L_{A}(a)^{2^{n}-2}\right)$.

In [38] and [37], two separate teams independently recovered the secret block cipher Chiasmus from an encryption tool called GSTOOL. Chiasmus is a German designed 64-bit block cipher which uses two S-boxes $S$ and $S^{-1}$. Schuster had the intuition that it was built similarly to the AES S-box. He was right. Using Lemma 1 and the linear equivalence algorithm of [4], we found that the S-box of Chiasmus is also based on a finite field inversion. However, unlike in the AES, it uses two affine mappings with non-zero constants. A script generating the S-box of Chiasmus is provided in the appendix of the full version of this paper [10]. The S-box itself can be found in a SAGE [19] module [36].

We could also have recovered this structure using directly the algorithm of Biryukov et al. [4] or the more recent one of Dinur [21]. However, the above approach and these algorithms share the same shortcoming when it comes to identifying the structure in an unknown S-box $F \in \mathfrak{S}_{2^{n}}$ : if we do not know the exact S-box to which $F$ might be affine-equivalent then they cannot be applied. Even if we know that it might be affine-equivalent to an SPN or a Feistel network, we cannot find the corresponding affine masks.

To solve this problem, we identify patterns in the LAT of the permutations with specific structures that are present regardless of the subfunctions they contain. As a consequence, they can always be detected.

\subsection{TU-Decomposition}

The TU-decomposition is a general structure that was first introduced in [7] where it was shown that the S-box of the latest Russian standards has such a structure. Later, it was encountered again in the context of the Big APN problem, a long standing open question in discrete mathematics. Indeed, the only known solution to this problem is a sporadic 6-bit APN permutation that was found by Dillon et al. [13] and which was proved in [35] to yield a TUdecomposition. This structure was then further decomposed to obtain the socalled open butterfly. As we will show below, some Feistel and SPN structures also share this decomposition. Thus, the tools that can find TU-decomposition can also be used to identify these structures even in the presence of affine masks.

Definition 2 ( $\mathrm{TU}_{t}$-decomposition). Let $n$ and $t$ be integers such that $0<t<$ $n$. We say that $F \in \mathfrak{S}_{2^{n}}$ has a $\mathrm{TU}_{t^{-}}$decomposition ${ }^{8}$ if there exists:

\footnotetext{
8 This is a simplified version of the $\mathrm{TU}_{t}$-decomposition compared to [15]. Indeed, in that paper, the authors only impose that $T_{y} \in \mathfrak{S}_{2^{n-t}} ; U_{x}$ may have collisions. Since we are only considering bijective S-boxes here, we consider that $U_{x} \in \mathfrak{S}_{2^{t}}$.
} 
- a family of $2^{n-t}$ permutations $T_{y} \in \mathfrak{S}_{2^{t}}$ indexed by $y \in \mathbb{F}_{2}^{n-t}$,

- a family of $2^{t}$ permutations $U_{x} \in \mathfrak{S}_{2^{n-t}}$ indexed by $x \in \mathbb{F}_{2}^{t}$, and

- two linear permutations $\mu: \mathbb{F}_{2}^{n} \rightarrow\left(\mathbb{F}_{2}^{t} \times \mathbb{F}_{2}^{n-t}\right)$ and $\eta:\left(\mathbb{F}_{2}^{t} \times \mathbb{F}_{2}^{n-t}\right) \rightarrow \mathbb{F}_{2}^{n}$

such that $F=\eta \circ G \circ \mu$, where $G$ is the permutation of $\mathbb{F}_{2}^{t} \times \mathbb{F}_{2}^{n-t}$ such that $G(x, y)=\left(T_{y}(x), U_{T_{y}(x)}(y)\right)$. This structure is presented in Figure 1a.

In other words, $F \in \mathfrak{S}_{2^{n}}$ has a $\mathrm{TU}_{t^{-}}$decomposition if and only if it is affineequivalent to $G \in \mathfrak{S}_{2^{n}}$ with the following property: if $G_{\uparrow t}$ is the restriction of $G$ to its $t$ bits of highest weight then $x \mapsto G_{\lceil t}(x \| a)$ is a permutation for all $a \in \mathbb{F}_{2}^{n-t}$.

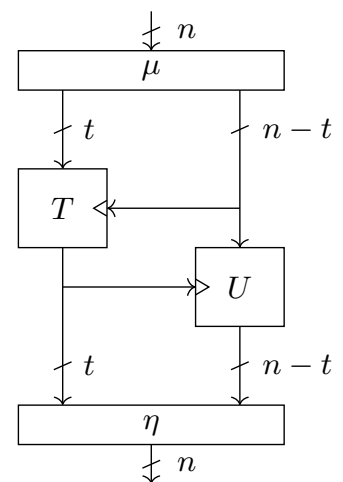

(a) $\mathrm{TU}_{t}$-decomposition.

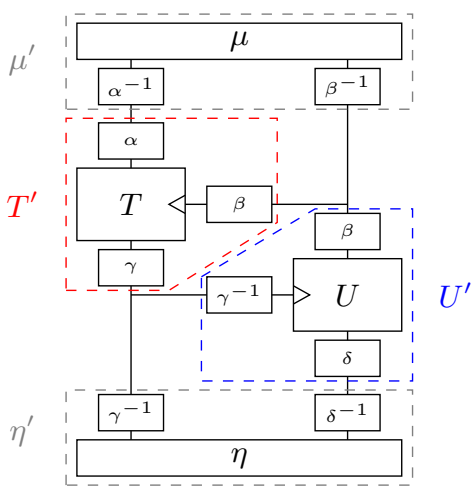

(b) Composing its components with linear permutations.

Fig. 1: Two functionally equivalent permutations.

Density of the set. In order to define a permutation with a $\mathrm{TU}_{t}$-decomposition, we need to choose $2^{n-t}$ permutations of $\mathfrak{S}_{2^{t}}, 2^{t}$ permutations of $\mathfrak{S}_{2^{n-t}}$ and two linear permutations operating on $n$ bits. However, several of the permutations generated in this way will be identical. Indeed, we can compose each $T_{y}$ with a $t$-bit linear permutation $\alpha \in \mathcal{L}_{2^{t}}$ to obtain a permutation $T_{y}^{\prime}=T_{y} \circ \alpha$. If we use $T_{y}^{\prime}$ and compose $\mu$ with $\alpha^{-1}$, then we obtain the same overall permutation as when $T_{y}$ and $\mu$ are used. More equivalent modifications can be made using linear permutations $\beta \in \mathcal{L}_{2^{n-t}}, \gamma \in \mathcal{L}_{2^{t}}$ and $\delta \in \mathcal{L}_{2^{n-t}}$, as summarized in Figure 1b. Hence, the total number of $n$-bit permutations with $\mathrm{TU}_{t}$-decompositions is at most

$$
\# \mathrm{TU}_{t} \leq \underbrace{\left|\mathfrak{S}_{2^{t}}\right|^{2^{n-t}}}_{T_{y}} \times \underbrace{\left|\mathfrak{S}_{2^{n-t}}\right|^{t}}_{U_{x}} \underbrace{\left(\frac{\left|\mathcal{L}_{2^{n}}\right|}{\left|\mathcal{L}_{2^{t}}\right| \times\left|\mathcal{L}_{2^{n-t}}\right|}\right)^{2}}_{\mu \text { and } \eta} .
$$

This quantity is only a bound as permutations that are self affine-equivalent lead to identical permutations with different $\mu$ and $\eta$. We used this bound to compute the anomaly associated to the presence of a $\mathrm{TU}_{t}$-decomposition in a permutation. It is given in Section 2 . 
How to recognize them? Let $F \in \mathfrak{S}_{2^{n}}$ be a permutation. As was established in Proposition 6 of [15], the presence of a $\operatorname{TU}_{t}$-decomposition is equivalent to the presence of a specific vector space of zeroes of dimension $n$ in $\mathcal{Z}_{F}$. Let us first recall the corresponding proposition in the particular case of permutations.

Proposition 4 ([15]). Let $F \in \mathfrak{S}_{2^{n}}$ and let $\mathcal{Z}_{F}$ be its Walsh zeroes. Then $F$ has a $\mathrm{TU}_{t}$-decomposition without any affine layers if and only if $\mathcal{Z}_{F}$ contains the vector space

$$
\left\{(0\|a, b\| 0), a \in \mathbb{F}_{2}^{t}, b \in \mathbb{F}_{2}^{n-t}\right\} .
$$

The advantage of Proposition 4 is that the pattern described depends only on the presence of a $\mathrm{TU}_{t}$-decomposition and not on the specifics of the components $T$ and $U$. Furthermore, recall that if $G=L_{2} \circ F \circ L_{1}$ for some linear permutations $L_{1}$ and $L_{2}$ then $\mathcal{W}_{G}(a, b)=\mathcal{W}_{F}\left(\left(L_{1}^{-1}\right)^{T}(a), L_{2}^{T}(b)\right)$.

Corollary 1. Let $F \in \mathfrak{S}_{2^{n}}$ and let $\mathcal{Z}_{F}$ be its Walsh zeroes. Then $F$ has a $\mathrm{TU}_{t^{-}}$ decomposition with linear permutations $\mu$ and $\eta$ if and only if

$$
\left\{\left(\left(\mu^{-1}\right)^{T}(0, a), \eta^{T}(b, 0)\right), a \in \mathbb{F}_{2}^{t}, b \in \mathbb{F}_{2}^{n-t}\right\} \subset \mathcal{Z}_{F}
$$

It is therefore sufficient to look for all the vector spaces of dimension $n$ contained in $\mathcal{Z}_{F}$ to see if $F$ has $\mathrm{TU}_{t}$-decomposition. If we find a vector space that is not the Cartesian product of a subspace of $\left\{(x, 0), x \in \mathbb{F}_{2}^{n}\right\}$ with a subspace of $\left\{(0, y), y \in \mathbb{F}_{2}^{n}\right\}$ then $F$ does not have a $\mathrm{TU}_{t}$-decomposition but there exists a linear function $L$ of $\mathbb{F}_{2}^{n}$ such that $F+L$ does [15]. Regardless, the key tool that allows the search for TU-decomposition is an efficient algorithm returning all the vector spaces of a given dimension that are contained in a set of elements of $\mathbb{F}_{2}^{n}$. Indeed, finding such vector spaces will allow us to recover all the values of $\left(\mu^{-1}\right)^{T}(0, a)$ and $\eta^{T}(b, 0)$ for $(a, b) \in \mathbb{F}_{2}^{t} \times \mathbb{F}_{2}^{n-t}$, from which we will deduce information about $\mu$ and $\eta$. We present such an algorithm in Section 4 and we used it as a subroutine of program finding a $\mathrm{TU}_{t}$-decomposition automatically (see the appendix of the full version [10]).

As observed in [15], the number of vector spaces of dimension $n$ in $\mathcal{Z}_{F}$ is the same as the number of vector spaces of dimension $n$ in the set of the coordinates of the zeroes in the DDT. Thus, we could equivalently present our results in terms of DDT.

\subsection{Substitution-Permutation Networks}

An $n$-bit SPN interleaves the parallel application of $k$ possibly distinct $m$-bit Sboxes with $n$-bit linear permutations, where $k \times m=n$. We use the common [8] notation $A S$ to denote a linear layer followed by an S-box layer. A $S A S$ structure is depicted in Figure 2a.

Let us estimate the number of $r$-round SPNs. As the S-box layers are interleaved with linear layers, we need to consider not the size of $\mathfrak{S}_{2^{m}}$ but instead the number of linear equivalence classes, which is at most $\left|\mathfrak{S}_{2^{m}}\right| /\left|\mathcal{L}_{2^{m}}\right|^{2}$. The 


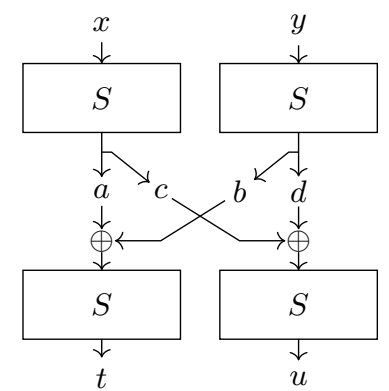

(a) A two-round SPN (SAS).

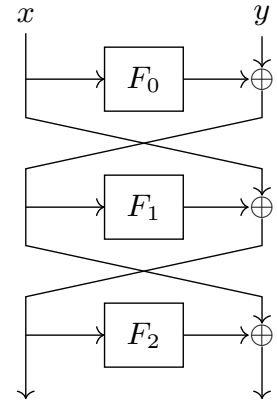

(b) A 3-round Feistel network.

Fig. 2: Two block-cipher-like S-box structures.

number of permutations with a $A(S A)^{r}$ structure is then at most

$$
\# A(S A)^{r} \leq\left(\frac{\left|\mathfrak{S}_{2^{m}}\right|}{\left|\mathcal{L}_{2^{m}}\right|^{2}}\right)^{r n / m} \times\left|\mathcal{L}_{2^{n}}\right|^{r+1}
$$

The corresponding anomalies for some values of $n$ are given in Section 3.5.

How to recognize them? First of all, the algebraic degree of a 2-round SPN is at most equal to $n-2$ [11]. Hence, if a permutation is of degree $n-1$, it cannot have such a structure.

In Theorem 3, we will establish the existence of specific vector space of zeroes in the LAT of a 2-round SPN. However, in order to properly state this theorem, we first need to introduce the following notion.

Definition 3 ( $m$-Valid minors). Let $k, m$ and $n$ be integers such that $n=$ $k \times m$. Let $L \in \mathcal{L}_{2^{n}}$ be a linear permutation. We define it using a $k^{2}$ block matrices $L_{i, j}$ of dimension $m \times m$ :

$$
L=\left[\begin{array}{ccc}
L_{0,0} & \ldots & L_{0, k-1} \\
\ldots & & \ldots \\
L_{k-1,0} & \ldots & L_{k-1, k-1}
\end{array}\right] .
$$

We call a minor of the matrix $L m$-valid if there exists a pair $I, J$ of subsets of $\{0, \ldots, k-1\}$ which are of the same size $0<|I|=|J|<k$ and such that the rank of $L_{I, J}=\left[L_{i, j}\right]_{i \in I, j \in J}$ is equal to $m$.

In other words, an $m$-valid minor of $L$ is a non-trivial minor of $L$ that is obtained by taking complete $m$-bit chunks of this matrix, and which has maximum rank.

Theorem 3. Let $F \in \mathfrak{S}_{2^{n}}$ be an $A S A S A$ structure built using $L$ as its central linear layer and two layers of $m$-bit $S$-boxes. For each $I, J \subsetneq\{0, \ldots, k-1\}$ defining an m-valid minor of $L$, there exists a vector space of zeroes of dimension $n$ in $\mathcal{Z}_{F}$. 
Proof. Because of Corollary 1, we restrict ourselves to the $S A S$ structure. If we let the input blocks corresponding to the indices in $I$ take all $2^{m}|I|$ possible values, then the output blocks with indices in $J$ will also take all $2^{m|J|}=2^{m|I|}$ possible values. There is thus a corresponding $\left.\mathrm{TU}_{m \mid I}\right|^{\text {-decomposition and hence }}$ a corresponding vector space in $\mathcal{Z}_{F}$.

This verification is less efficient than the dedicated cryptanalysis methods presented in [30]. However, the aim here is not so much to recover the ASASA structure used, it is rather to identify the S-box as having such a structure in the first place. Using the following corollary, we can immediately understand why $N_{V}=\left(\begin{array}{c}2 \times 2 \\ 2\end{array}\right)=6$ for several S-boxes in Table 1: it is a direct consequence of their 2-round SPN structure and of the strong diffusion of their inner linear layer.

Corollary 2. Let $F \in \mathfrak{S}_{2^{n}}$ be the $S A S$ structure built using $L$ as its linear layer and two layers of $m$-bit $S$-boxes, where $n=k \times m$. If $L$ is $M D S$ over the alphabet of $S$-box words, then $\mathcal{Z}_{F}$ contains at least $\left(\begin{array}{c}2 k \\ k\end{array}\right)$ vector spaces of dimension $n$.

Proof. As $L$ is MDS, all its minors and in particular those corresponding to the definition of $m$-minors have a maximum rank. There are $\sum_{i=1}^{k}\left(\begin{array}{c}k \\ i\end{array}\right) \times\left(\begin{array}{c}k \\ i\end{array}\right)$ such $m$-minors, to which we add the "free" vector space $\left\{(x, 0), x \in \mathbb{F}_{2}^{n}\right\}$ which is always present: there are at least $\sum_{i=0}^{k}\left(\begin{array}{c}k \\ i\end{array}\right)^{2}=\left(\begin{array}{c}2 k \\ k\end{array}\right)$ vector spaces in $\mathcal{Z}_{F}$.

\section{$3.4 \quad$ Feistel Networks}

The Feistel structure is a classical block cipher construction which is summarized in Figure 2b. The number of permutations that are affine-equivalent to $r$-round Feistel networks that use permutations as the round functions is at most equal to

$$
\underbrace{\left|\mathfrak{S}_{2^{n / 2}}\right|^{r}}_{\text {round funcs. }} \times \underbrace{\frac{1}{\left(2^{n}\right)^{\left\lceil\frac{n}{2}\right\rceil}}}_{\text {constants }} \times \underbrace{\left|\mathcal{L}_{2^{n}}\right|^{2}}_{\text {outer layers }} \times \underbrace{\frac{1}{\left|\mathcal{L}_{2^{n / 2}}\right|^{2}}}_{\text {branch transforms }}
$$

Indeed, we can apply $n / 2$-bit linear permutations $L$ and $L^{\prime}$ to each branch and, provided that the round functions are modified, we can cancel them out by applying $L^{-1}$ and $\left(L^{\prime}\right)^{-1}$ on the output branches. We can also add constants freely to the output of the first $\lceil r / 2\rceil$ round functions, as explained in [5].

How to recognize them? There are efficient function-recovery techniques for up to 5-round Feistel networks [5]. However, as soon as affine masks are added, the corresponding techniques can no longer be applied. Still, as with the SPN structure, Feistel networks with few rounds exhibit specific vector spaces in their Walsh zeroes as was already observed for 4-round Feistel network in [7]. This means that it is possible to detect such structures using the vector spaces in their Walsh zeroes.

Theorem 4 ([7]). Let $F$ be a 4-round Feistel network such that round functions 2 and 3 are permutations. Then $\mathcal{W}_{F}(x\|y, 0\| y)=0$ for all $x, y$ in $\mathbb{F}_{2}^{n / 2}$. 
This observation also holds for a 3-round Feistel. In fact, there are more vector spaces in such a structure.

Theorem 5. Let $F_{0}, F_{1}$ and $F_{2}$ be functions of $\mathbb{F}_{2}^{n / 2}$ such that $F_{1} \in \mathfrak{S}_{2^{n / 2}}$. Let $F \in \mathbb{F}_{2}^{n}$ be the 3-round Feistel network using $F_{0}, F_{1}$ and $F_{2}$ as its round functions. Then the set $\mathcal{Z}_{F}$ contains the following vector spaces of dimension $n$ :

1. $\left\{(x, 0), x \in \mathbb{F}_{2}^{n}\right\},\left\{(0, y), y \in \mathbb{F}_{2}^{n}\right\}$,

2. $\left\{(x\|0, y\| 0),(x, y) \in \mathbb{F}_{2}^{n / 2} \times \mathbb{F}_{2}^{n / 2}\right\}$,

3. $\left\{(x\|y, x\| 0),(x, y) \in \mathbb{F}_{2}^{n / 2} \times \mathbb{F}_{2}^{n / 2}\right\},\left\{(x\|0, x\| y),(x, y) \in \mathbb{F}_{2}^{n / 2} \times \mathbb{F}_{2}^{n / 2}\right\}$,

4. $\left\{(x\|y, 0\| y),(x, y) \in \mathbb{F}_{2}^{n / 2} \times \mathbb{F}_{2}^{n / 2}\right\},\left\{(0\|y, x\| y),(x, y) \in \mathbb{F}_{2}^{n / 2} \times \mathbb{F}_{2}^{n / 2}\right\}$,

the fourth category being present if $F_{0}$ and $F_{2}$ are in $\in \mathfrak{S}_{2^{n / 2}}$.

The proof of this theorem follows from direct applications of results in [15] and of these observations:

- if the 3-round Feistel network implies a specific vector space, it also implies the one with the coordinates swapped because its inverse is also a 3-round Feistel network,

- $(x, y) \mapsto F(x, y) \oplus(x, 0)$ is a permutation if $F_{1} \in \mathfrak{S}_{2^{n / 2}}$, and

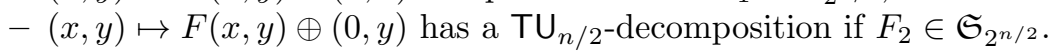

The details are provided in the appendix of the full version [10].

\subsection{Structural Anomalies}

In light of our results, we can quantify the anomaly associated to the presence of various structures. In this case, the mapping $P$ considered maps $\mathfrak{S}_{2^{n}}$ to $\{0,1\}$ : a permutation has a specific structure or it does not. The anomaly associated to a given structure is then

$$
\mathrm{A}_{\text {structure }}=-\log _{2}\left(\frac{\mid\left\{G \in \mathfrak{S}_{2^{n}}, G \text { has the structure }\right\} \mid}{\left|\mathfrak{S}_{2^{n}}\right|}\right),
$$

meaning that the set sizes we extracted above allow us to quantify the anomalies associated to the $\mathrm{TU}_{t}$-decomposition, the SPN structure, the Feistel network and the TKlog (see below for the latter). The corresponding anomalies are summarized in Table 2 for different values of $n$.

The existence of a TU-decomposition with $t=1$ for $F \in \mathfrak{S}_{2^{n}}$ is equivalent to the presence of a component with a linear structure [15], i.e. to the existence of $a \in \mathbb{F}_{2}^{n}$ such that the Boolean function $x \mapsto a \cdot F(x)$ has a probability 1 differential. Thus, the corresponding row of Table 2 gives the anomaly corresponding to linear structures.

We can also compute the anomaly associated to the TKlog structure [34] used in the S-box of Streebog and Kuznyechik $[22,23]$ called $\pi \in \mathfrak{S}_{2^{8}}$. A TKlog is a $2 m$-bit permutation parametrized by an affine function $\kappa: \mathbb{F}_{2}^{m} \rightarrow \mathbb{F}_{2^{2 m}}$ such 
that $\kappa(x)=\Lambda(x) \oplus \kappa(0)$ for some linear function $\Lambda$. This function must be such that $\operatorname{Im}(\Lambda) \cup \mathbb{F}_{2^{m}}$ spans $\mathbb{F}_{2^{2 m}}$. The TKlog also depends on a permutation $s$ of $\mathfrak{S}_{2^{m}-1}$. It is defined as follows

$$
\begin{cases}\pi(0) & =\kappa(0) \\ \pi\left(\alpha^{\left(2^{m}+1\right) j}\right) & =\kappa\left(2^{m}-j\right), \text { for } 1 \leq j<2^{m}-1 \\ \pi\left(\alpha^{i+\left(2^{m}+1\right) j}\right) & =\kappa\left(2^{m}-i\right)+\alpha^{\left(2^{m}+1\right) s(j)}, \text { for } i<2^{m}+1, j<2^{m}-1\end{cases}
$$

where $\alpha$ is a root of a primitive polynomial $p$ of degree $2 m$, so that $\alpha^{2^{m}+1}$ is a multiplicative generator of $\mathbb{F}_{2^{m}}^{*}$. The number of TKlog, is then given by

$$
\underbrace{\prod_{i=m}^{2 m-1}\left(2^{2 m}-2^{i}\right)}_{\Lambda} \times \underbrace{\left|\mathfrak{S}_{2^{m}-1}\right|}_{s} \times \underbrace{\left(\phi\left(2^{2 m}-1\right) /(2 m)\right)}_{\# \text { primitive polynomials }} \times \underbrace{2^{2 m}}_{\kappa(0)}
$$

where $\phi$ is Euler's totient function. As for the inverse function, the encoding of the elements of $\mathbb{F}_{2^{2 m}}$ as binary strings can be considered to be part of the outer affine layers.

\begin{tabular}{ccrrrr}
\hline Structure. & Parameters & $n=6$ & $n=8$ & $n=12$ & $n=16$ \\
\hline$x \mapsto x^{2^{n}-2}$ & - & 236.1 & 1570.6 & 42981.2 & 953548.5 \\
\hline \multirow{2}{*}{ TKlog } & "pure" & 258.7 & 1601.5 & 42870.7 & 952207.7 \\
& $\mathrm{AE}$ & 184.3 & 1469.0 & 42574.2 & 951683.2 \\
\hline \multirow{2}{*}{ TU-dec. } & $t=1$ & 8.8 & 95.7 & 1997.7 & 32699.7 \\
& $t=n / 2$ & 13.0 & 201.1 & 5215.3 & 91571.2 \\
\hline \multirow{2}{*}{ SPN } & ASASA, $S / r=2$ & 192.7 & 1435.4 & 41913.5 & 947036.0 \\
& ASASASA, $S / r=2$ & 158.2 & 1342.3 & 41316.3 & 943662.7 \\
\hline \multirow{2}{*}{ Feistel } & 3-round, $F_{i} \in \mathfrak{S}_{2^{n / 2}}$ & 205.5 & 1443.3 & 41898.2 & 946980.9 \\
& 4-round, $F_{i} \in \mathfrak{S}_{2^{n / 2}}$ & 220.8 & 1487.6 & 42194.2 & 948664.9 \\
\hline
\end{tabular}

Table 2: Upper bounds on the anomalies of the affine-equivalence to some structures. For the TKlog, "AE" corresponds to permutations affine-equivalent to some TKlog and "pure" to TKLog themselves. $S / r$ is the number of S-boxes used in each round, i.e. the number that are applied in parallel.

\section{Vector Spaces Extraction Algorithms}

Let $\mathcal{S}$ be a set of elements of $\mathbb{F}_{2}^{n}$. In this section, we describe an algorithm which extracts all the vector spaces of dimension at least $d$ that are completely 
contained in $\mathcal{S}$. As established in the previous section, the ability to solve this problem will allow us to identify TU-decompositions, some SPNs, and 3,4-round Feistel networks even in the presence of affine encodings. It can also test the CCZ-equivalence [16] of a function to a permutation, as was done by Dillon et al. [13] to find the first APN permutation operating on an even number of bits.

Our results can be interpreted using both the ordering relation over the integers and by reasoning over the respective position of the zeroes of the elements in $\mathbb{F}_{2}^{n}$. The following lemma links these two views.

Definition 4 (Most Significant Bit). Let $x \in \mathbb{F}_{2}^{n}$ and let us write $x=$ $(x[0], \ldots, x[n-1])$ where $x[0]$ is the least significant bit. We denote $M S B(x)$ the greatest index $i$ such that $x[i]=1$.

Lemma 2. For any $x \in \mathbb{F}_{2}^{n}$, it holds that

$$
x<x \oplus a \Leftrightarrow x[M S B(a)]=0,
$$

where the order relation is obtained by interpreting $x$ and $x \oplus a$ as the binary representations of integers.

\subsection{A Simple Approach and How Ours Improves It}

Let us first present a naive approach to solving this problem. At its core, this approach is a tree search that builds the complete vector spaces iteratively.

Starting from a specific element $x \in \mathcal{S}$ and vector space $V_{x}=\{0, x\}$, we loop over all the elements $y$ such that $y>x$ and check whether $(x \oplus y) \in \mathcal{S}$, in which case we build $V_{x, y}=V_{x} \cup\left\{y \oplus v, v \in V_{x}\right\}$. We then repeat this process by looking for $z>y$ such that $(z \oplus v) \in \mathcal{S}$ for all $v \in V_{x, y}$. This process can then be iterated until complete bases $(x, y, z, \ldots)$ of vector spaces are found. Our approach is based on the same principles but it significantly outperforms this naive algorithm by solving its two main shortcomings.

First, the basis of a vector space is not unique. The condition that it be ordered, which is implied by the algorithm sketched above, is not sufficient to ensure uniqueness. This implies that the algorithm will be slowed down by the exploration of the branches that actually correspond to identical spaces, and that a post processing checking for duplicated spaces will be needed. Our algorithm will solve this problem and return exactly one basis for each vector space contained in $\mathcal{S}$. These bases are called Gauss-Jordan Bases (GJB) and are introduced in Section 4.2.

Second, at each iteration, we need to consider all $z \in \mathcal{S}$ such that $z$ is strictly larger than the largest vector already in the basis being built. In our approach, we update at each iteration a set that contains all the elements $z$ that could be used to construct a larger basis using a process which we call vector extraction (see Section 4.3). Like in the algorithm above, this set only contains elements that are strictly greater than the previous bases elements. However, it is also strictly larger than all the elements in the vector space spanned by this basis and its size is reduced by at least a factor 2 at each iteration. Using vector 
extractions, we can also skip the test that $(z \oplus v) \in \mathcal{S}$ for all $v$ in the current vector space which will increase the speed of our algorithm.

Besides, in each iteration, we use a heuristic method to consider only a subset of this set of $z$ which is based on the number and positions of its zeroes, the Bigger $M S B$ Condition.

In summary, we improve upon the algorithm above in the following ways:

- we construct exactly one basis per vector space contained in $\mathcal{S}$ (using GJB, see Section 4.2),

- we significantly reduce the number of vectors that can be considered in the next iterations (using vector extractions, see Section 4.3), and

- we further decrease the number of vectors that need to be explored at a given iteration using a specific filter (using the Bigger MSB condition, see Section 4.4).

Finally, the vector space extraction algorithm itself is presented in Section 4.5. An algorithm extracting affine spaces which uses the former as a subroutine is presented along with an actual implementation of the vector space algorithm in the appendix of the full version [10].

In [14], Canteaut et al. introduced an algorithm which, given an $n$-bit Boolean function $f$, lists all the affine spaces of dimension $m$ such that $f$ is constant (or affine) on them. Our algorithm can easily perform the same task. Indeed, $f$ is affine on a subspace $U$ if and only if $\{x \| f(x), x \in U\}$ is an affine subspace, meaning that our affine space search algorithms can list all such spaces.

Using our implementation (see [10]), we only need about 12 min to reprove their Fact 22 which deals with a 14-bit Boolean function while they claim a runtime of $50 \mathrm{~h}$ in this case. Our machine is more recent and thus likely faster than theirs but not by a factor 250: our algorithm is inherently more efficient. It is also far more versatile, as we have established above.

\subsection{Gauss-Jordan Bases}

These objects are those which our vector space search will actually target. They were described in the context of Boolean functions in [14].

Definition 5 (GJB [14]). For any vector space $V$ of dimension d, the GaussJordan Basis (GJB) of $V$ is the set $\left\{v_{0}, \ldots, v_{d-1}\right\}$ such that $\left\langle v_{0}, \ldots, v_{d-1}\right\rangle=V$ which is the smallest such set when sorted in lexicographic order.

For any space $V$ there is exactly one GJB. Indeed, we can write down all of its bases, sort the elements in each of them in increasing order and then sort the reordered bases in lexicographic order. This implies that $v_{i}<v_{i+1}$ for all $i$. Some key properties of GJBs are given by the following lemma.

Lemma 3. GJBs have the following properties.

1. If $\left\{v_{0}, \ldots, v_{i}\right\}$ is the GJB of $\left\langle v_{0}, \ldots, v_{i}\right\rangle$ then $\left\{v_{0}, \ldots, v_{i-1}\right\}$ is a GJB. 
2. The basis $\left\{v_{0}, \ldots, v_{d-1}\right\}$ is a GJB if and only if

$$
\left\{\begin{array}{l}
\forall j \in\{0, \ldots, d-2\}, M S B\left(v_{j}\right)<M S B\left(v_{j+1}\right) \\
\forall i \in\{1, \ldots, d-1\}, \forall j \in\{0, \ldots, i-1\}, v_{i}\left[M S B\left(v_{j}\right)\right]=0 .
\end{array}\right.
$$

3. If $\left\{v_{0}, \ldots, v_{d-1}\right\}$ is a GJB then, for all $j \in\{0, \ldots, d-1\},\left\langle v_{0}, \ldots, v_{d-1}\right\rangle$ contains exactly $2^{j}$ elements $x$ such that $M S B(x)=M S B\left(v_{j}\right)$.

Proof. We prove each point separately.

Point 1. A basis of $\left\langle v_{0}, \ldots, v_{i-1}\right\rangle$ lexicographically smaller than $\left\{v_{0}, \ldots, v_{i}\right\}$ could be used to build a basis of $\left\langle v_{0}, \ldots, v_{i}\right\rangle$, lexicographically smaller than its GJB, which is impossible.

Point 2. We prove each direction of the equivalence separately.

$\Rightarrow$ Suppose that $\left\{v_{0}, \ldots, v_{d-1}\right\}$ is indeed a GJB. Then $\operatorname{MSB}\left(v_{j}\right)=\operatorname{MSB}\left(v_{j+1}\right)$ would imply that $\operatorname{MSB}\left(v_{j} \oplus v_{j+1}\right)<\operatorname{MSB}\left(v_{j}\right)$ which, in particular, would imply that $v_{j} \oplus v_{j+1}<v_{j}$. This would contradict that $\left\{v_{0}, \ldots, v_{d-1}\right\}$ is a GJB. Similarly, $\operatorname{MSB}\left(v_{j}\right)>\operatorname{MSB}\left(v_{j+1}\right)$ would imply $v_{j}>v_{j+1}$ which is also a contradiction. We deduce that $\operatorname{MSB}\left(v_{j}\right)<\operatorname{MSB}\left(v_{j+1}\right)$ for any $0 \leq j<d-1$. Suppose now that $v_{i}\left[\operatorname{MSB}\left(v_{j}\right)\right]=1$ for some $j<i$. We deduce from Lemma 2 that $v_{i} \geq v_{i} \oplus v_{j}$, which is again a contradiction because $\left\{v_{0}, \ldots, v_{d-1}\right\}$ is minimal. We have thus established that if $\left\{v_{0}, \ldots, v_{d-1}\right\}$ is a GJB then it must satisfy the conditions in Equation (4).

$\Leftarrow$ Let us now assume that these conditions hold. In this case, we have that $v_{i}<v_{i} \oplus \bigoplus_{j \in I} v_{j}$ for any subset $I$ of $\{0, \ldots, i-1\}$ because the MSB of $\bigoplus_{j \in I} v_{j}$ is always strictly smaller than $\operatorname{MSB}\left(v_{i}\right)$ and because of Lemma 2. Thus, adding $v_{i}$ at the end of $\left\{v_{0}, \ldots, v_{i-1}\right\}$ yields a GJB of $\left\langle v_{0}, \ldots, v_{i}\right\rangle$. A simple induction then gives us the result.

Point 3. Using the first point of this lemma allows us to proceed via a simple induction over the size of the basis. If the basis is simply $\left\{v_{0}\right\}$ then the lemma obviously holds. Then, adding an element $v_{d}$ to the end of a GJB of size $d$ will add $2^{d}$ elements $x$ such that $\operatorname{MSB}(x)=\operatorname{MSB}\left(v_{d}\right)$.

The last point of Lemma 3 allows a significant speed up of the search for such GJBs. To describe it, we introduce the following concept.

Definition 6 (MSB spectrum). Let $\mathcal{S}$ be a set of elements in $\mathbb{F}_{2}^{n}$. The MSB spectrum of $\mathcal{S}$ is the sequence $\left\{N_{i}(\mathcal{S})\right\}_{0 \leq i<n}$ such that

$$
N_{i}(\mathcal{S})=\#\{x \in \mathcal{S}, \operatorname{MSB}(x)=i\} .
$$

Corollary 3 (MSB conditions). If a set $\mathcal{S}$ of elements from $\mathbb{F}_{2}^{n}$ contains a vector space of dimension $d$, then there must exist a strictly increasing sequence $\left\{m_{j}\right\}_{0 \leq j \leq d-1}$ of length $d$ such that

$$
N_{m_{j}}(S) \geq 2^{j} .
$$




\subsection{Vector Extractions}

We now present a class of functions called extractions which will play a crucial role in our algorithms. We also prove their most crucial properties.

Definition 7 (Extraction). Let $a \neq 0$ be some element of $\mathbb{F}_{2}^{n}$. The extraction of $a$, denoted $\mathcal{X}_{a}$, is a function mapping a subset $\mathcal{S}$ of $\mathbb{F}_{2}^{n}$ to $\mathcal{X}_{a}(\mathcal{S})$, where $x \in$ $\mathcal{X}_{a}(\mathcal{S})$ if and only if all of the following conditions are satisfied:

$$
x \in \mathcal{S}, \quad(x \oplus a) \in \mathcal{S}, \quad a<x<(x \oplus a) .
$$

In particular, $\mathcal{X}_{a}(\mathcal{S}) \subseteq \mathcal{S}$. Our algorithm will iterate such extractions to construct smaller and smaller sets without loosing any GJBs. This process is motivated by the following theorem.

Theorem 6. Let $\left\{v_{0}, \ldots, v_{i-1}\right\}$ be elements of some subset $\mathcal{S}$ of $\mathbb{F}_{2}^{n}$ such that $0 \in \mathcal{S}$ and such that $v_{j+1} \in\left(\mathcal{X}_{v_{j}} \circ \ldots \circ \mathcal{X}_{v_{0}}\right)(\mathcal{S})$ for all $j<i$. Then it holds that $v_{i} \in\left(\mathcal{X}_{v_{i-1}} \circ \ldots \circ \mathcal{X}_{v_{0}}\right)(\mathcal{S})$ if and only if $\left\langle v_{0}, \ldots, v_{i}\right\rangle \subseteq \mathcal{S}$ and $\left\{v_{0}, \ldots, v_{i}\right\}$ is the $G J B$ of this vector space.

Proof. In order to prove the theorem, we proceed by induction over $i$ using the validity of the theorem over bases of size $i$ as our induction hypothesis. At step $i$, we assume that $v_{0}, \ldots, v_{i}$ are elements of $\mathcal{S}$ and that $v_{j+1} \in\left(\mathcal{X}_{v_{j}} \circ \ldots \circ \mathcal{X}_{v_{0}}\right)(\mathcal{S})$ for all $j<i$.

Initialization $\mathbf{i}=\mathbf{1}$. By definition of vector extraction, $v_{1} \in \mathcal{X}_{v_{0}}(\mathcal{S})$ if and only if $v_{1} \in \mathcal{S}$, and $v_{0} \oplus v_{1} \in \mathcal{S}, v_{0}<v_{1}<v_{0} \oplus v_{1}$. As we assume $0, v_{0} \in \mathcal{S}$, this is equivalent to $\left\{0, v_{0}, v_{1}, v_{0} \oplus v_{1}\right\}=\left\langle v_{0}, v_{1}\right\rangle$ being contained in $\mathcal{S}$ and to $\left\{v_{0}, v_{1}\right\}$ being a GJB.

Inductive Step $\mathbf{i}>\mathbf{1}$ Let $v_{i} \in\left(\mathcal{X}_{v_{i-1}} \circ \ldots \circ \mathcal{X}_{v_{0}}\right)(\mathcal{S})$. From the induction hypothesis, we have that $\left\{v_{0}, \ldots, v_{i-1}\right\}$ is a GJB. Using the second point of Lemma 3 , we have that its extension $\left\{v_{0}, \ldots, v_{i}\right\}$ is a GJB if and only if $v_{i}\left[\operatorname{MSB}\left(v_{j}\right)\right]=0$ (which is equivalent to $v_{i}<v_{i} \oplus v_{j}$ ) for all $0 \leq j<i$ and $\operatorname{MSB}\left(v_{i}\right)>\operatorname{MSB}\left(v_{i-1}\right)$.

By definition of $\mathcal{X}_{v_{j}}$, we have that $v_{i}<v_{i} \oplus v_{j}$ for all $j$ such that $0 \leq j<$ $i$, so $\left\{v_{0}, \ldots, v_{i}\right\}$ is a GJB if and only if $\operatorname{MSB}\left(v_{i}\right)>\operatorname{MSB}\left(v_{i-1}\right)$. We have $v_{i-1}<v_{i}<v_{i} \oplus v_{i-1}$, which implies in particular $v_{i-1}<v_{i} \oplus v_{i-1}$, so that $v_{i}\left[\operatorname{MSB}\left(v_{i-1}\right)\right]=0$. Thus, $v_{i}>v_{i-1}$ holds if and only if $\operatorname{MSB}\left(v_{i}\right)>$ $\operatorname{MSB}\left(v_{i-1}\right)$.

Corollary 4. If $\left\{e_{0}, \ldots, e_{d-1}\right\}$ is the GJB of a vector space $V$ such that $V \subseteq$ $\mathcal{S} \subseteq \mathbb{F}_{2}^{n}$ then, for all $0<j \leq d-1$, we have

$$
\left\langle e_{j}, e_{j+1}, \ldots, e_{d-1}\right\rangle \subseteq\left(\mathcal{X}_{e_{j-1}} \circ \ldots \mathcal{X}_{e_{1}} \circ \mathcal{X}_{e_{0}}\right)(\mathcal{S})
$$

Evaluating $\mathcal{X}_{a}$ imposes a priori to look whether $x \oplus a$ belongs in $\mathcal{S}$ for all $x \in \mathcal{S}$ such that $x<x \oplus a$. This verification can be implemented efficiently using a binary search when $\mathcal{S}$ is sorted. We can make it even more efficient using the following lemma. 
Lemma 4. Let $\mathcal{S}$ be a set of elements in $\mathbb{F}_{2}^{n}$ and let $a \in \mathcal{S}$. Then we have

$$
\mathcal{X}_{a}(\mathcal{S})=\bigcup_{i=M S B(a)+1}^{n} \mathcal{X}_{a}(\{x \in \mathcal{S}, M S B(x)=i\})
$$

\subsection{Bigger MSB Condition}

The following lemma provides a necessary condition for some $e_{0} \in \mathcal{S}$ to be the first element of a GJB of size $d$.

Lemma 5 (Bigger MSB condition). If $e_{0}$ is the first element in a GJB of size $d$ of elements of a set $\mathcal{S}$ of elements in $\mathbb{F}_{2}^{n}$, then $\mathcal{S}^{\prime}$ defined as

$$
\mathcal{S}^{\prime}=\left\{x \in \mathcal{S}, \operatorname{MSB}(x)>\operatorname{MSB}\left(e_{0}\right)\right\}
$$

must satisfy the MSB condition of Corollary 3 for dimension d-1, i.e. there is a strictly increasing sequence $\left\{m_{j}\right\}$ of length $d-1$ such that

$$
\#\left\{x \in \mathcal{S}, \operatorname{MSB}(x)=m_{j}\right\}>2^{j} .
$$

This lemma provides an efficient filter to know whether $x$ can be the start of a GJB of size $d$ which depends only on the MSB of $x$, so that it does not need to be evaluated for all $x \in \mathcal{S}$ but only once for each subset of $\mathcal{S}$ with a given MSB.

\subsection{Vector Space Extraction Algorithm}

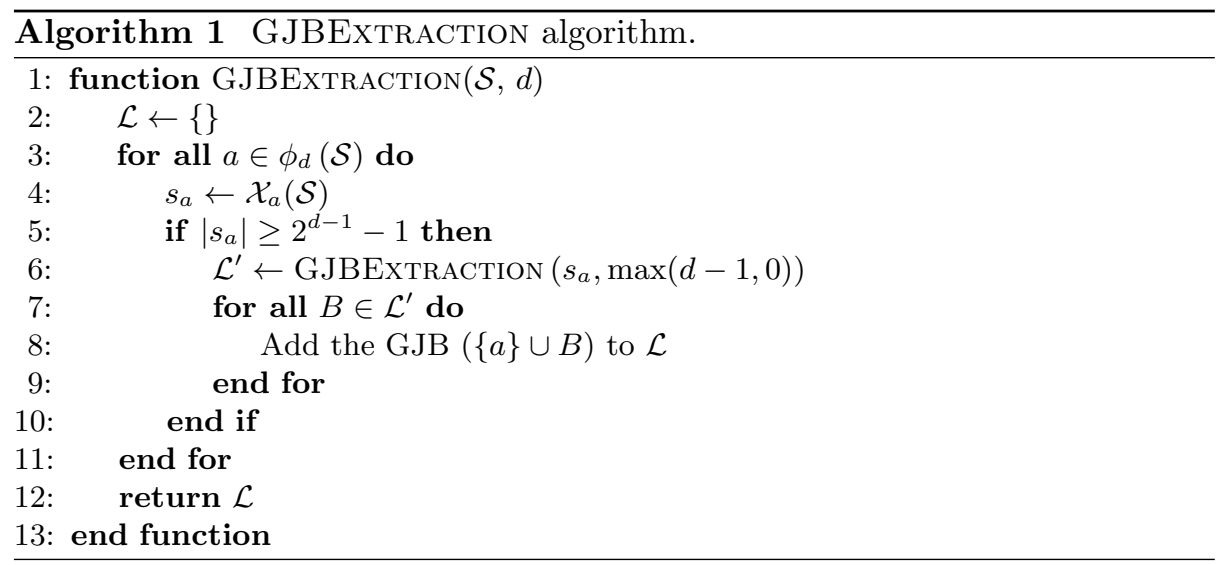

If we let $\phi_{d}$ be the identity then we can directly deduce from Theorem 6 and Corollary 4 that GJBExtRaction (as described in Algorithm 1) returns the unique GJBs of each and every vector space of dimension at least equal to $d$ that is included in $\mathcal{S}$. 
This algorithm can be seen as a tree search. The role of $\phi_{d}$ is then to cut branches as early as possible by allowing us to ignore elements that cannot possibly be the first element of a base of size $d$ by implementing the Bigger MSB Condition of Lemma 5:

$$
a \in \phi_{d}(\mathcal{S}) \text { if and only if } \exists\left\{m_{j}\right\}_{0 \leq j<d},\left\{\begin{array}{l}
m_{j+1}>m_{j}>\operatorname{MSB}(a) \\
\#\left\{x \in \mathcal{S}, \operatorname{MSB}(x)=m_{j}\right\}>2^{j}
\end{array}\right.
$$

Note that we only need to try and build such a sequence of increasing $m_{j}$ once for each value of $\operatorname{MSB}(x)$ for $x \in \mathcal{S}$. It is possible to check for the existence of such a sequence in a time proportional to $|\mathcal{S}|$.

\section{Conclusion}

We have presented a comprehensive list of anomalies quantifying how unlikely the properties of a given S-box are. These can be of a statistical nature and we have pioneered the use of the BCT for this purpose. They can also correspond to the presence of a specific structure, many of which are particular cases of the TU-decomposition. To find TU-decompositions, we presented an efficient vector space algorithm which can be of independent interest. We have also showed how finding TU-decompositions can help bypass affine masks for several S-box structures.

We can apply our results to $\pi$, the 8-bit S-box used by both Streebog [22] and Kuznyechik [23]. It has very high anomalies (see Table 3) which means that the set of S-boxes with as strong a structure as the TKlog found in $\pi$ is very small. This observation is coherent with the claim of [34] that the structure of $\pi$ was deliberately inserted by its designers.

\begin{tabular}{|c|c|c|c|c|}
\hline \multicolumn{3}{|c|}{ Statistical } & \multicolumn{2}{|c|}{ Structural } \\
\hline Differential & Linear & Boomerang & $\mathrm{TU}_{4}$ & TKlog \\
\hline $80.6^{\dagger}$ & 34.4 & 14.2 & 201.1 & 1601.5 \\
\hline
\end{tabular}

We finally list some open problems that we have identified while working on this paper.

Open Problem 1. How can we better estimate the differential anomaly?

Open Problem 2. Why are there so many vector spaces in $\mathcal{Z}_{F}$ when $F$ is a 3 -round Feistel network of $\mathfrak{S}_{2^{8}}$ ? 


\section{Acknowledgement}

We thank Jérémy Jean for shepherding this paper. We also thank Florian Wartelle for fruitful discussions about vector space search, and Anne Canteaut for proofreading a first draft of this paper. The work of Xavier Bonnetain receives funding from the European Research Council (ERC) under the European Union's Horizon 2020 research and innovation programme (grant agreement no. $714294-$ acronym QUASYModo). The work of Shizhu Tian is supported by the National Science Foundation of China (No. 61772517, 61772516).

\section{References}

1. Advanced Encryption Standard (AES). National Institute of Standards and Technology (NIST), FIPS PUB 197, U.S. Department of Commerce (Nov 2001)

2. Biham, E., Shamir, A.: Differential cryptanalysis of DES-like cryptosystems. In: Menezes, A.J., Vanstone, S.A. (eds.) CRYPTO'90. LNCS, vol. 537, pp. 2-21. Springer, Heidelberg (Aug 1991)

3. Biham, E., Shamir, A.: Differential cryptanalysis of Feal and N-hash. In: Davies, D.W. (ed.) EUROCRYPT'91. LNCS, vol. 547, pp. 1-16. Springer, Heidelberg (Apr 1991)

4. Biryukov, A., De Canniére, C., Braeken, A., Preneel, B.: A toolbox for cryptanalysis: Linear and affine equivalence algorithms. In: Biham, E. (ed.) EUROCRYPT 2003. LNCS, vol. 2656, pp. 33-50. Springer, Heidelberg (May 2003)

5. Biryukov, A., Leurent, G., Perrin, L.: Cryptanalysis of Feistel networks with secret round functions. In: Dunkelman, O., Keliher, L. (eds.) SAC 2015. LNCS, vol. 9566, pp. 102-121. Springer, Heidelberg (Aug 2016)

6. Biryukov, A., Perrin, L.: On reverse-engineering S-boxes with hidden design criteria or structure. In: Gennaro, R., Robshaw, M.J.B. (eds.) CRYPTO 2015, Part I. LNCS, vol. 9215, pp. 116-140. Springer, Heidelberg (Aug 2015)

7. Biryukov, A., Perrin, L., Udovenko, A.: Reverse-engineering the S-box of streebog, kuznyechik and STRIBOBr1. In: Fischlin, M., Coron, J.S. (eds.) EUROCRYPT 2016, Part I. LNCS, vol. 9665, pp. 372-402. Springer, Heidelberg (May 2016)

8. Biryukov, A., Shamir, A.: Structural cryptanalysis of SASAS. In: Pfitzmann, B. (ed.) EUROCRYPT 2001. LNCS, vol. 2045, pp. 394-405. Springer, Heidelberg (May 2001)

9. Blondeau, C., Canteaut, A., Charpin, P.: Differential properties of $x \mapsto x^{2^{t}-1}$. IEEE Transactions on Information Theory 57(12), 8127-8137 (2011)

10. Bonnetain, X., Perrin, L., Tian, S.: Anomalies and vector space search: Tools for S-box analysis (full version). Cryptology ePrint Archive, Report 2019/528 (2019), https://eprint.iacr.org/2019/528

11. Boura, C., Canteaut, A.: On the influence of the algebraic degree of $f^{-1}$ on the algebraic degree of $g \circ f$. IEEE Transactions on Information Theory 59(1), 691-702 (Jan 2013)

12. Boura, C., Perrin, L., Tian, S.: Boomerang uniformity of popular S-box constructions. In: WCC 2019: The Eleventh International Workshop on Coding and Cryptography (2019) 
13. Browning, K.A., Dillon, J., McQuistan, M.T., Wolfe, A.J.: An APN permutation in dimension six. In: Post-proceedings of the 9-th International Conference on Finite Fields and Their Applications. vol. 518, pp. 33-42. American Mathematical Society (2010)

14. Canteaut, A., Daum, M., Dobbertin, H., Leander, G.: Finding nonnormal bent functions. Discrete Applied Mathematics 154(2), 202 - 218 (2006), coding and Cryptography

15. Canteaut, A., Perrin, L.: On CCZ-equivalence, extended-affine equivalence, and function twisting. Finite Fields and Their Applications 56, 209-246 (2019)

16. Carlet, C., Charpin, P., Zinoviev, V.: Codes, bent functions and permutations suitable for DES-like cryptosystems. Designs, Codes and Cryptography 15(2), 125$156(1998)$

17. Cid, C., Huang, T., Peyrin, T., Sasaki, Y., Song, L.: Boomerang connectivity table: A new cryptanalysis tool. In: Nielsen, J.B., Rijmen, V. (eds.) EUROCRYPT 2018, Part II. LNCS, vol. 10821, pp. 683-714. Springer, Heidelberg (Apr / May 2018)

18. Daemen, J., Rijmen, V.: Probability distributions of correlation and differentials in block ciphers. Journal of Mathematical Cryptology 1(3), 221-242 (2007)

19. Developers, T.S.: SageMath, the Sage Mathematics Software System (Version 7.5.1) (2017), http://www.sagemath.org

20. Diffie, W., (translators), G.L.: SMS4 encryption algorithm for wireless networks. Cryptology ePrint Archive, Report 2008/329 (2008), http://eprint.iacr.org/ 2008/329

21. Dinur, I.: An improved affine equivalence algorithm for random permutations. In: Nielsen, J.B., Rijmen, V. (eds.) EUROCRYPT 2018, Part I. LNCS, vol. 10820, pp. 413-442. Springer, Heidelberg (Apr / May 2018)

22. Federal Agency on Technical Regulation and Metrology: Information technology - data security: Hash function. English version available at http://wwwold.tc26.

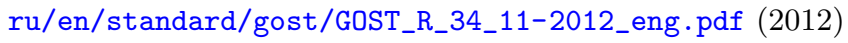

23. Federal Agency on Technical Regulation and Metrology: Information technology - data security: Block ciphers. English version available at http://wwwold.tc26.

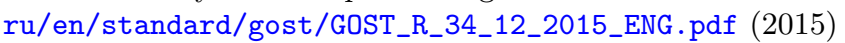

24. Helleseth, T. (ed.): EUROCRYPT'93, LNCS, vol. 765. Springer, Heidelberg (May 1994)

25. Kazymyrov, O., Kazymyrova, V., Oliynykov, R.: A method for generation of highnonlinear s-boxes based on gradient descent. Cryptology ePrint Archive, Report 2013/578 (2013), http://eprint.iacr.org/2013/578

26. Li, K., Qu, L., Sun, B., Li, C.: New results about the boomerang uniformity of permutation polynomials. CoRR abs/1901.10999 (2019), http://arxiv.org/abs/ 1901.10999

27. Liu, F., Ji, W., Hu, L., Ding, J., Lv, S., Pyshkin, A., Weinmann, R.P.: Analysis of the SMS4 block cipher. In: Pieprzyk, J., Ghodosi, H., Dawson, E. (eds.) ACISP 07. LNCS, vol. 4586, pp. 158-170. Springer, Heidelberg (Jul 2007)

28. Lupanov, O.B.: On Networks Consisting of Functional Elements with Delays, pp. 43-83. Springer US, New York, NY (1973), https://doi.org/10.1007/ 978-1-4757-0079-4_3

29. Matsui, M.: Linear cryptanalysis method for DES cipher. In: Helleseth [24], pp. 386-397

30. Minaud, B., Derbez, P., Fouque, P.A., Karpman, P.: Key-recovery attacks on ASASA. Journal of Cryptology 31(3), 845-884 (Jul 2018)

31. Nyberg, K.: Differentially uniform mappings for cryptography. In: Helleseth [24], pp. $55-64$ 
32. O'Connor, L.: On the distribution of characteristics in bijective mappings. In: Helleseth [24], pp. 360-370

33. O'Connor, L.: Properties of linear approximation tables. In: Preneel, B. (ed.) FSE'94. LNCS, vol. 1008, pp. 131-136. Springer, Heidelberg (Dec 1995)

34. Perrin, L.: Partitions in the S-box of Streebog and Kuznyechik. IACR Trans. Symm. Cryptol. 2019(1), 302-329 (2019)

35. Perrin, L., Udovenko, A., Biryukov, A.: Cryptanalysis of a theorem: Decomposing the only known solution to the big APN problem. In: Robshaw, M., Katz, J. (eds.) CRYPTO 2016, Part II. LNCS, vol. 9815, pp. 93-122. Springer, Heidelberg (Aug 2016)

36. Perrin, L., Wiemer, F.: S-Boxes used in cryptographic schemes. Available online at https://git.sagemath.org/sage.git/tree/src/sage/crypto/sboxes.py (2017)

37. Schejbal, J., Tews, E., Wälde, J.: Reverse engineering of chiasmus from gstool. Presentation at the Chaos Computer Club (CCC). (2013)

38. Schuster, F.: Reverse engineering of chiasmus from gstool. Presentation at the HGI-Kolloquium. Slides available at https://prezi . com/ehrz4krw2z0d/hgi-chm/ (January 2014)

39. Shannon, C.E.: The synthesis of two-terminal switching circuits. The Bell System Technical Journal 28(1), 59-98 (Jan 1949)

40. Tardy-Corfdir, A., Gilbert, H.: A known plaintext attack of FEAL-4 and FEAL6. In: Feigenbaum, J. (ed.) CRYPTO'91. LNCS, vol. 576, pp. 172-181. Springer, Heidelberg (Aug 1992)

41. Wagner, D.: The boomerang attack. In: Knudsen, L.R. (ed.) FSE'99. LNCS, vol. 1636, pp. 156-170. Springer, Heidelberg (Mar 1999) 